\title{
The High Definition Earth Viewing (HDEV) Payload
}

\author{
Paul Muri \\ NASA Johnson Space \\ Center \\ 2101 NASA Parkway \\ Houston, TX 77058 \\ 281-483-8341 \\ paul.muri@nasa.gov
}

\author{
Susan Runco \\ NASA Johnson Space \\ Center \\ 2101 NASA Parkway \\ Houston, TX 77058 \\ 281-244-8848 \\ susan.k.runco@nasa.gov
}

\author{
Carlos Fontanot \\ NASA Johnson Space \\ Center \\ 2101 NASA Parkway \\ Houston, TX 77058 \\ 281-483-2398 \\ carlos.fontanot-1@nasa.gov
}

\author{
Chris Getteau \\ NASA Johnson Space Center \\ 2101 NASA Parkway \\ Houston, TX 77058 \\ 281-244-7359 \\ christian.getteau-1@nasa.gov
}

\begin{abstract}
The High Definition Earth Viewing (HDEV) payload enables long-term experimentation of four, commercial-of-theshelf (COTS) high definition video, cameras mounted on the exterior of the International Space Station. The payload enables testing of cameras in the space environment. The HDEV cameras transmit imagery continuously to an encoder that then sends the video signal via Ethernet through the space station for downlink. The encoder, cameras, and other electronics are enclosed in a box pressurized to approximately one atmosphere, containing dry nitrogen, to provide a level of protection to the electronics from the space environment. The encoded video format supports streaming live video of Earth for viewing online. Camera sensor types include chargecoupled device and complementary metal-oxide semiconductor. Received imagery data is analyzed on the ground to evaluate camera sensor performance. Since payload deployment, minimal degradation to imagery quality has been observed. The HDEV payload continues to operate by live streaming and analyzing imagery. Results from the experiment reduce risk in the selection of cameras that could be considered for future use on the International Space Station and other spacecraft. This paper discusses the payload development, endto-end architecture, experiment operation, resulting image analysis, and future work.
\end{abstract}

\section{TABLE OF Contents}

1. INTRODUCTION ......................................................... 1

2. RADIATION ANALYSIS BACKGROUND................... 2

3. CONCEPT OF OPERATIONS ................................... 3

4. SYSTEM DESIGN.................................................... 4

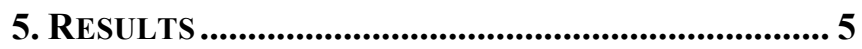

6. CONCLUSIONS AND FUTURE WORK ...................... 5

REFERENCES.............................................................. 6

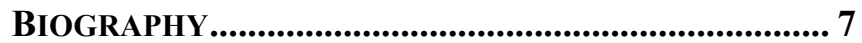

\section{INTRODUCTION}

For video camera equipment in previous space exploration programs, vendors would be contracted to design a specialized cameras to survive the harsh radiation and temperature elements endured. The development time and cost were very high for these customized cameras and rigs. These customized mission cameras were active for short periods, an order of weeks, with exposure to a space environment [1]. As a result, the International Space Station (ISS) program set out to experiment with the design, installation, and experimentation of a payload with completely commercial cameras and imagery processing equipment, and to create a payload enclosure to sustain nominal operations of commercial cameras.
The payload was called High Definition Earth Viewing, also known as HDEV, and started as a Technology Demonstration effort within the ISS Payloads Office in April 2011. The project goals were to create a payload with multiple external high definition (HD) cameras to mount on the exterior of the ISS with Commercial Off The Shelf (COTS) hardware. The experiment goals were evaluate the longevity of COTS cameras to the space environment, and secondarily to provide HD video of Earth to the public.

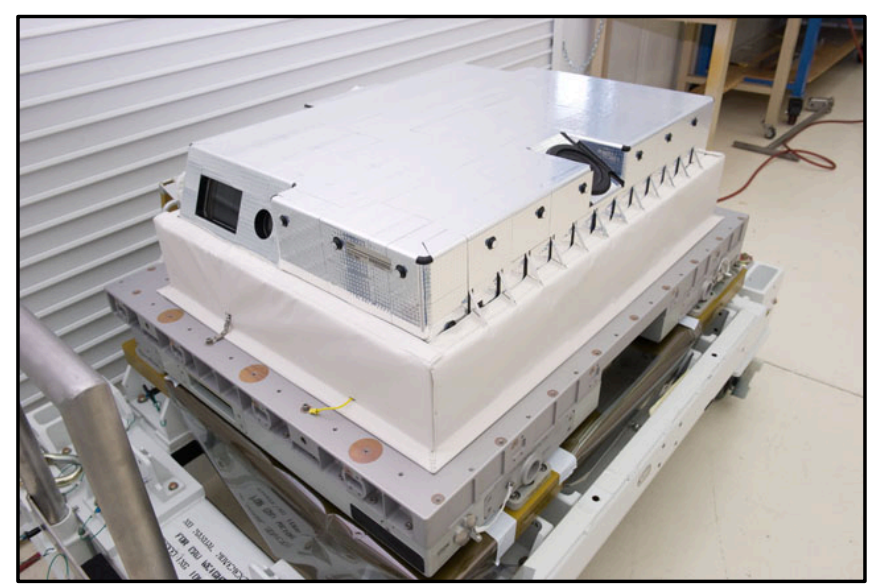

Figure 1. HDEV's assembled payload

The final HDEV payload consisted of just a single enclosure shown in Figure 1. The cameras are enclosed in a temperature specific housing. High school students helped design some of the cameras' components, through the High Schools United with NASA to Create Hardware (HUNCH) program, and student teams operate the experiment. The four cameras chosen were from Panasonic, Sony, Hitachi and Toshiba. The camera properties are listed in Table 1. The cameras sensors were either charge-coupled device (CCD) or complementary metal-oxide semiconductor (CMOS).

Table 1. Commercial HDEV Camera Models

\begin{tabular}{|l|l|}
\hline \multicolumn{1}{|c|}{ Camera } & \multicolumn{1}{c|}{ Sensor Type } \\
\hline \hline & \\
Panasonic AG-HMC150 & $1 / 3$ inch CCD (x3) \\
Sony FCB-EH4300 & $1 / 3$ inch CMOS, 2 megapixel \\
Toshiba IK-HR1S & $1 / 3$ inch CMOS, 2.1 megapixel \\
Hitachi HV-HD30 & $1 / 3$ inch CMOS, 1.3 megapixel \\
& \\
\hline
\end{tabular}


The HDEV payload was designed, built, and ready for shipment to Kennedy Space Center for launch by March 2012. The payload's rapid design to flight hardware build was attributed to utilizing of COTS hardware. COTS hardware could be used because there were fewer requirements on this payload not being mission critical to the ISS and crew.

Despite the quick design and payload manufacturing, the payload was not placed on a manifest until the launch of SpaceX Cargo Resupply (CRS) 3, two years later. HDEV was launched on SpaceX CRS-3 on April 2014. The wait time was due to numerous changes in launch schedule. Once delivered to the ISS, HDEV was quickly installed and activated just 12 days later on April 30, 2014.

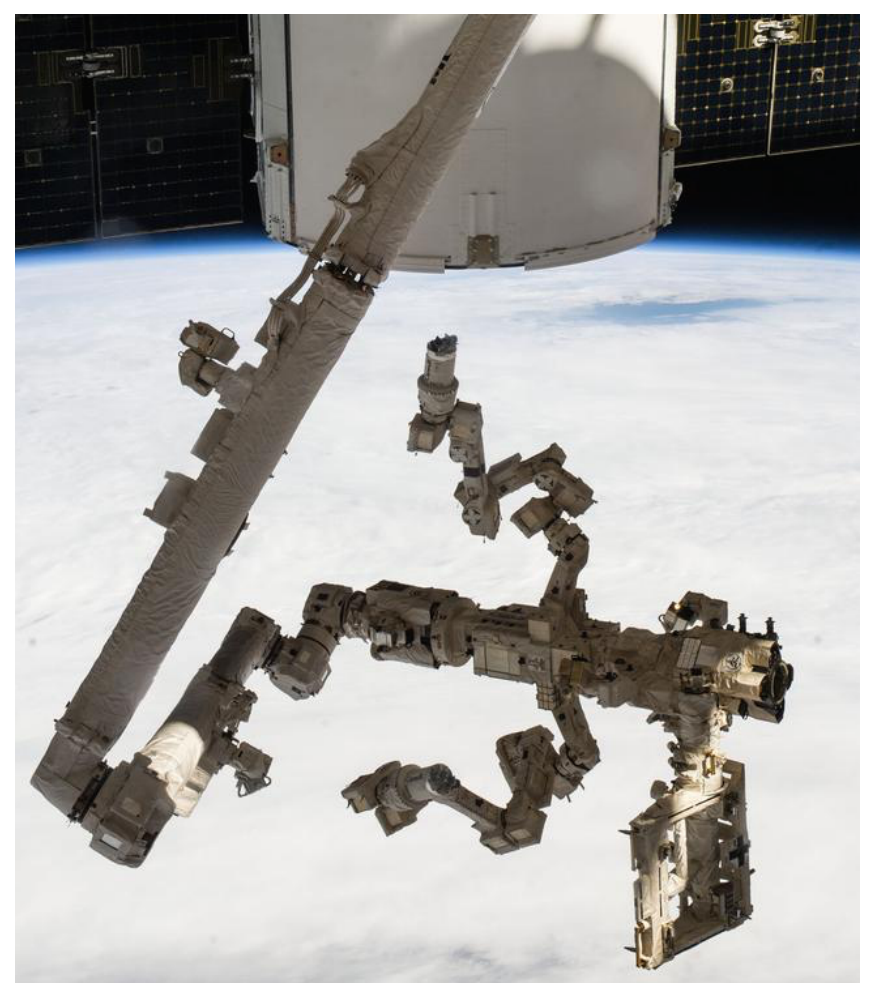

Figure 2. Robotic ground controllers used the Canadarm2 and Dexter to remove the HDEV payload from the trunk of the SpaceX Dragon

The HDEV installation was notable because robotic transfer from launch vehicle's trunk to external payload operational site installed the payload. Therefore, no scheduled Extravehicular activity (EVA) or Extra Vehicular Robotics (EVR) maintenance requirements was required for this external payload, and no ISS crew interaction was required.

The Special Purpose Dexterous Manipulator, the Canadian Space Agency's robotic handyman, also known as Dexter, and the Canadarm2 or Space Station Remote Manipulator System arm removed the payload, shown in Figure 2 from the trunk to mount it on the nadir adapter of the European Space Agency's Columbus exposed facility shown in Figure 3 . Using an automated robotic arm as automated process was carried out for the first time in the ISS program with the installation of the HDEV payload [2].

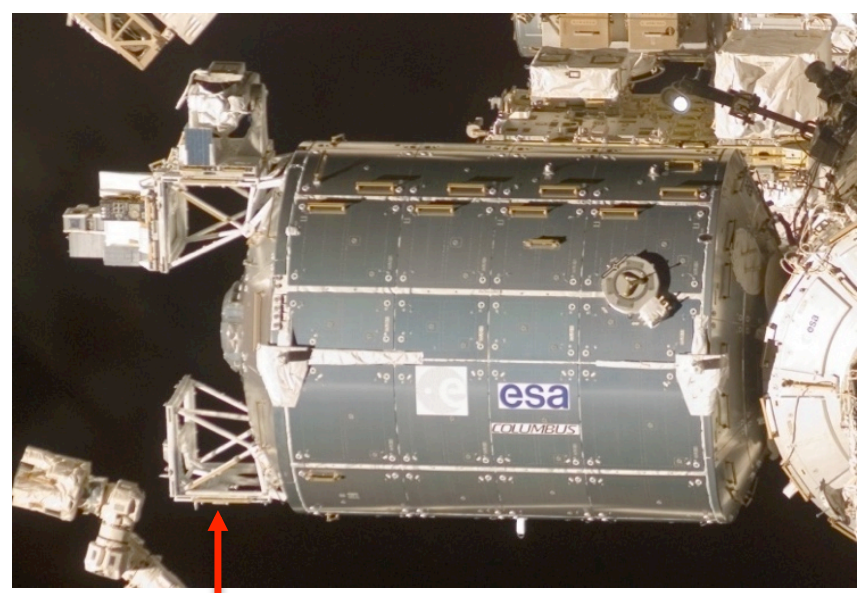

HDEV installation location

Figure 3. The mounting location for HDEV on ESA Columbus

Until HDEV, the International Space Station (ISS) flew with standard definition external cameras, and internal HD cameras, which downlinked a combination of six independent video streams during end-to-end signal connections. Now with HDEV installed, the ISS had four HD cameras continuously operating on the exterior with no required crew intereaction.

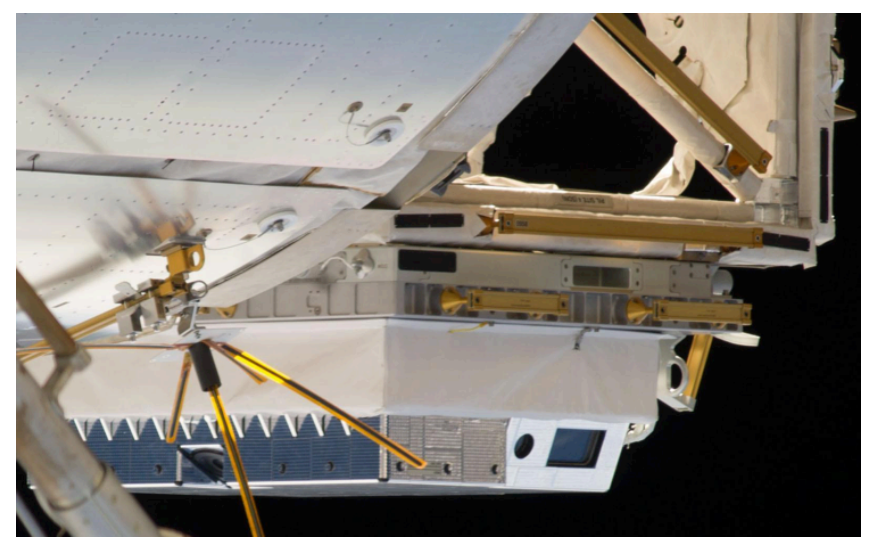

Figure 4. The HDEV payload Mounted

The rest of this paper is organized as follows: a brief background on radiation analysis in section 2. Section 3 describes the concept of operations for HDEV. Section 4 provides a detailed systems design of the payload, components, dataflow, and experiment. Section 5 describes the results thus far. Last, section 6 presents the experiment conclusions, and future work.

\section{RADIATION ANALYSIS BACKGROUND}

HDEV monitors the rate at which HD video camera image quality degrades when exposed to the space environment (mainly from cosmic radiation damage) and verifies the 
effectiveness of the design of the HDEV housing for thermal control.

There are two main types of radiation effects Total Ionization Dose (ITD), and Single Event Effect (SEE):

1. Total Ionization Dose is damage from protons or electron depositing energy in material by ionization. For active electronics, this effect causes a shift of the threshold voltage, leakage current, gain variation, and reduced noise immunity.

2. Single Event Effect is the transient effect by high-energy particles to semiconductors causing an abnormal response, such as a burn or short, in the circuit.

For ITD, aluminum shielding enclosure can be used to mitigate radiation exposure. However, in the case of SEE the shielding would not prevent high energy penetrating particles. As a result, active electronics can provide redundancy if nominal electron charges are affected [3].

\section{CONCEPT OF OPERATIONS}

The four cameras are pointed in three difference directions: the Hitachi camera points forward, the Sony and Panasonic cameras point aft, and Toshiba points nadir (down towards Earth) as shown in Figure 5.

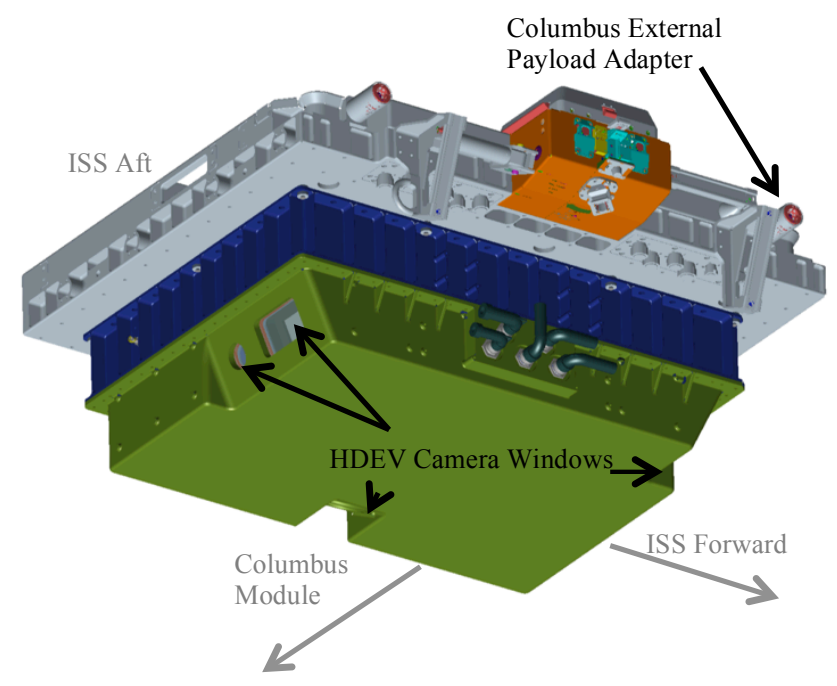

Figure 5. Nadir View Model

HDEV operates one camera at a time. HDEV is designed so that when the system is initially powered on, after a 2minute start up period, the cameras are turned on one at a time in a repeating cycle. The forward camera is powered first, followed by the nadir and then each aft looking camera, such that the HDEV video "follows" a location on the earth as the ISS passes overhead. This auto-cycle mode of the HDEV does not require any input from ground operators, so the HDEV can be operated any time that the ISS power and data resources are available, without requiring a ground controller present to operate the payload.
The payload imaging team provided a formula to sequence each camera for the best possible view. It depended on the angle each camera was facing. If the cycle of the four cameras were not carefully timed, in some instances, cameras would pick up extreme sun glare or reflections through the payload window.

Alternately as desired by ground controllers, HDEV video can be commanded. Ground operators have the choice to change the cycle of the images noted in the auto-cycle mode (either changing which cameras that are powered on, or changing the length of time they are powered on), or, if desired, ground controllers can command a single camera to remain powered on and no auto-cycle to take place.

The software for controlling HDEV has following capabilities:

- Issue commands to the HDEV Hardware through Huntsville Operations Support Center.

- Select camera or cycle through video

- Setup camera switching Cycle

- View HDEV health message status

- Record health status (Pressure/Temp, Voltage, Current) message for later analysis

Since the station orbits the earth once every 90 minutes, it experiences a sunrise or a sunset about every 45 minutes. When the station is in darkness, external camera video may appear black, but can sometimes provide spectacular views of lightning or city lights below.

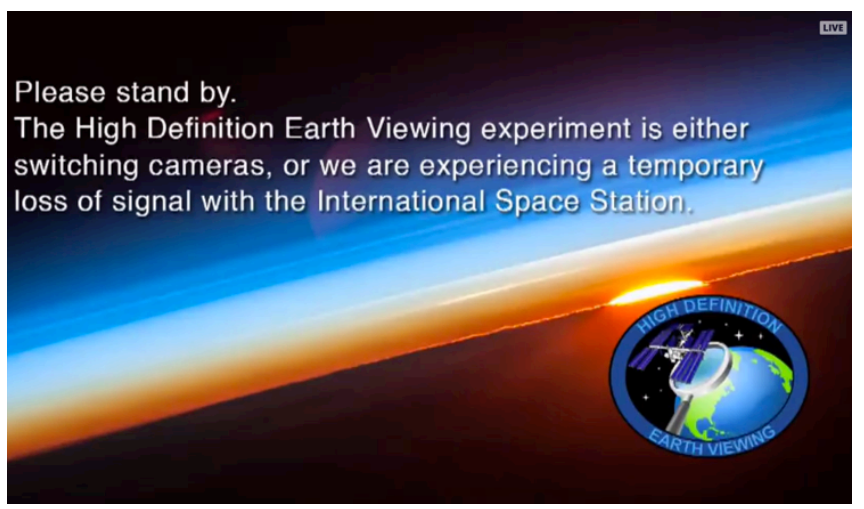

Figure 6. LOS or Camera Cycle Notification

Live streaming video is only available when the space station is in contact with the ground. During "loss of signal" (LOS) or a camera cycle period, viewers will see a notification to please stand by as shown in Figure 6 .

The HDEV video cameras are a fixed payload camera system that requires no zoom, no pan or tilt mechanisms. The video imagery is encoded into an Ethernet compatible format for transmission to the ground and further distribution. In this format, the video can be viewed from any computer connected to the Internet. 
HDEV does not record video on board the ISS, all video is transmitted to the ground real time; any desired recording of the video occurs as ground operations.

\section{SYSTEM DESIGN}

\section{Payload Design}

The entire payload is enclosed in a pressurized box to provide a level of protection to the electronics from the space environment. The enclosure contains dry nitrogen at Atmospheric pressure.

In addition to the four cameras, the payload's integrated Command and Data Handling (C\&DH) avionics (Ethernet), and power/data distribution circuit boards allow the integration of the payload's components interface to the ISS Columbus module. HDEV avionics enclosed include the following: VSI ANV443 Encoder (configured for 720p60 output per NASA standard; 6Mbps nominal data rate), AJA HA5 to convert HDMI to HD-SDI, two power distribution circuit Boards, and an Extron SW4 3G HD-SDI Router. The enclosure components are diagramed in Figure 7. The Camera switching is done via National Instruments CompactRio Single Board Computer (SBC) Module.

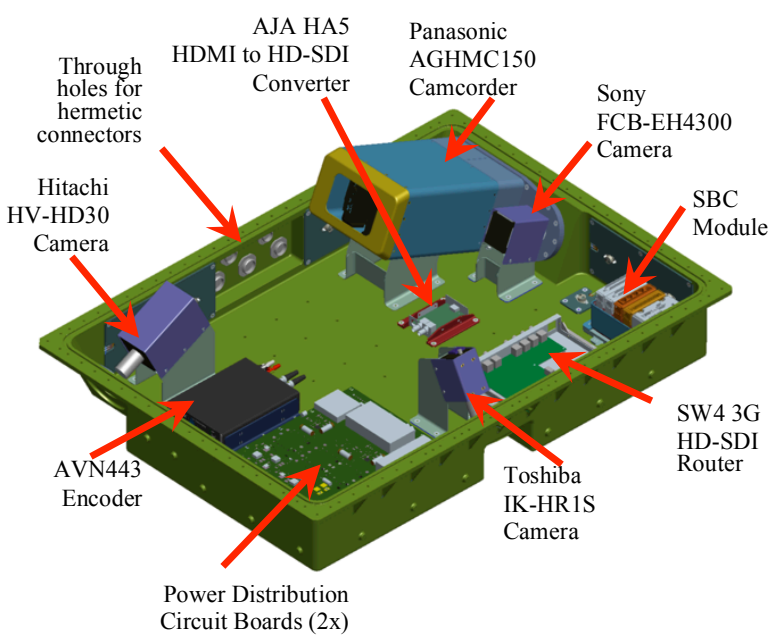

Figure 7. HDEV Payload Electronics Model

The CompactRio reads commands via 1553 data handling. Single Event Latch circuitry incorporated for the cameras, SBC, encoder, router, and converter. Cameras are monitored and controlled by SBC. Latch-up circuit enables selfshutdown and recovery.

\section{Optics}

The HDEV cameras use a mix of various lens to provide a proper frame without pan and tilt capabilities needed. The Hitachi and Toshiba cameras use a Fujinon TF4DA-8 4mm F/2.2 C-mount Wide Angle Lens. The Panasonic uses an included Leica Dicomar lens w/13x zoom, F/1.6-3.0. The Sony included a lens with the shortest focal length is 4.7 $\mathrm{mm}$. The optics provide for approximately a $500 \mathrm{~m}$ spatial resolution [4]. Temporal resolution can be between 180 minutes and three days when taking into account LOS and nights [4].

\section{System Architecture}

The cameras are connected via the SBC switch to a video encoder. This encoder converts the video to a H.264 video stream, which then feeds to a ISS computer. This computer adds the CCSDS headers required by the station's network. The signal is then downlinked via Tracking and Data Relay Satellite (TDRS) to the ground segment at White Sands New Mexico.

The ground segment starts in White Sands, New Mexico, which forwards the data to the Payload Operations Integration Center (POIC) at Marshall Spaceflight Center, in Huntsville, AL [5].

Using the Telescience Resource Kit (TReK), the POIC depacketizes CCSDS data, then sends the data to Johnson Space Center (JSC) in Houston, TX to create a public realtime transport protocol (RTP) stream and archive video when required. A simplified data flow diagram is shown in Figure 8 [5].

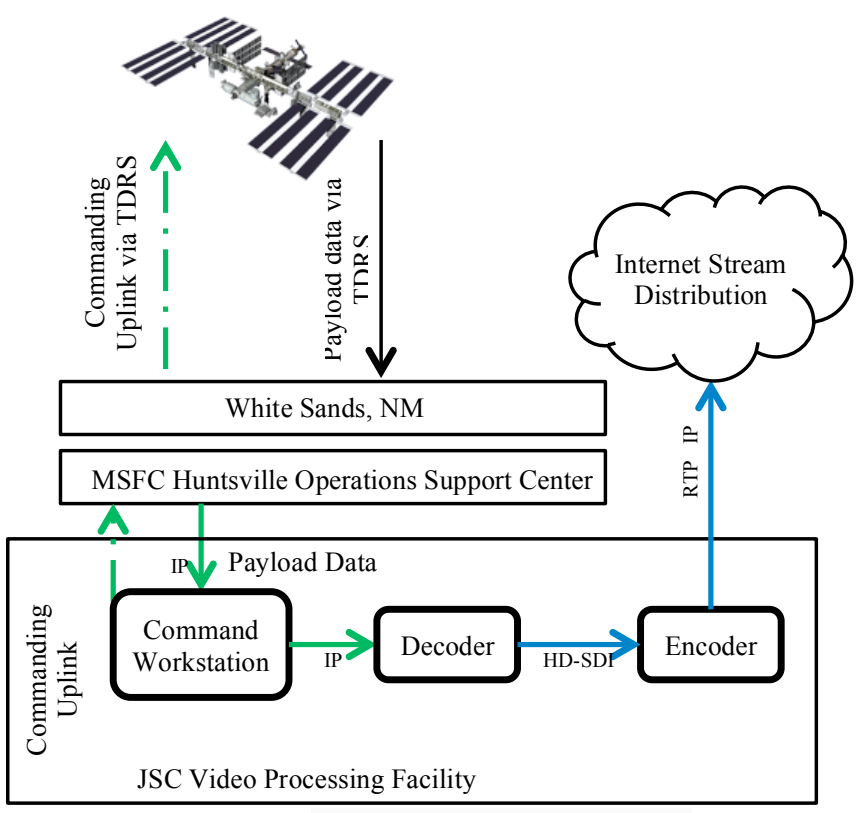

Figure 8. HDEV data flow diagram

\section{Pixel Radiation Experiment}

During night passes, there is black on the screen $(\sim 45$ minutes). During the time when the payload can be command, and video is recorded on the ground to provide pixel analysis. The pixel analysis video of taken at night, when there is a flat color, then run through pixel correction software to determine a pixel recorded as off color. Scanning for pixels is done on a monthly basis. During night passes, there is very little visibility (blackness) on the screen for about 45 minutes. 


\section{RESULTS}

Pixel Experiment

As of 2016, the pixel correction software has reported less than 30 damaged pixels from the four cameras.

Table 2. Pixel Corrective Scan as of October 2016

\begin{tabular}{|l|c|}
\hline \multicolumn{1}{|c|}{ Camera } & $\begin{array}{c}\text { Pixel Corrections } \\
\text { Required }\end{array}$ \\
\hline \hline Panasonic AG-HMC150 & 15 \\
Sony FCB-EH4300 & 0 \\
Toshiba IK-HR1S & 1 \\
Hitachi HV-HD30 & 12 \\
\hline
\end{tabular}

The first visual confirmation of a bad pixel occurred in August 2014. The pixel appeared in nadir camera (Toshiba). Visibility of the pixel depends on surrounding information, brightness. This pixel damage was not visible during night passes. Inconclusive results are due to number of factors including inconsistent times for camera operations.

The HDEV video is encoded on the payload using a VSI AVN-443 encoder. This encoder is the same model that is used for ISS internal video. Given that HDEV video is encoded as an h.264 stream, $720 \mathrm{p}$ at $6 \mathrm{Mbps}$, the compression, and data rate of the encoder also affects the resolution, which can mask bad pixels.

Hardware voltage stays within specification parameter and about 1 watt is utilized for each active device. Typical temperature is $22 \mathrm{C}$ and pressure is approximately $12 \mathrm{PSI}$ (close to 1 atmosphere).

Public Viewing

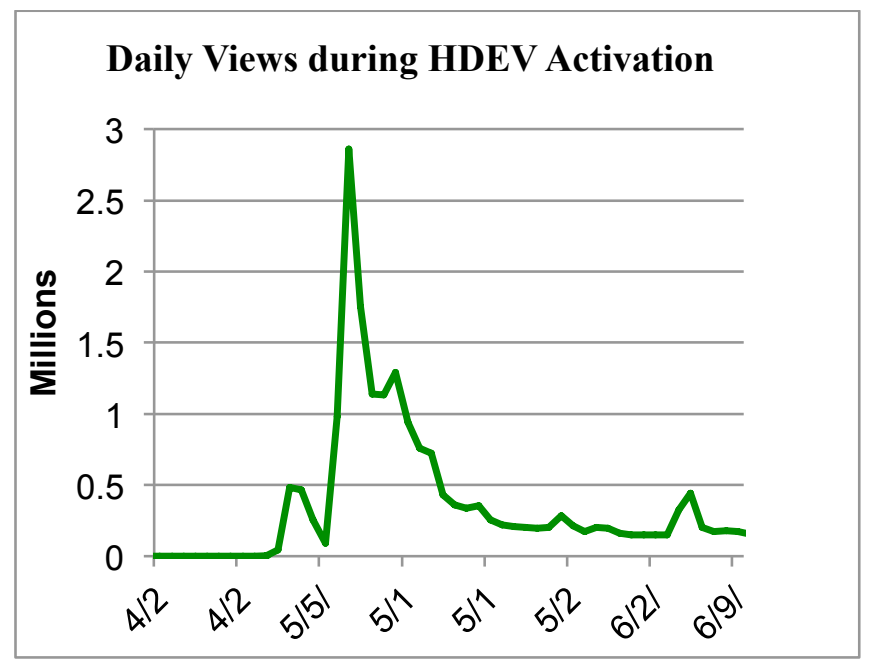

Figure 9. Daily view from Ustream channel during HDEV activation
One week after HDEV's official activation, the payload Ustream viewing went through an ephemeral viral sensation. This was due to many news outlets, and Internet blogs reporting on the activation of the payload [6]. On May 7th 2014, HDEV received 2.8 million views for the day. That daily peak subsided quickly, however there is still a steady number of viewers that visit the Ustream site to watch. As of October 2016, there are over 92 million views, according to the Ustream counter [7].

There are also notable peak view increases during natural events that are viewable. In particular, the weather channel has also used live views of Hurricanes from HDEV to show a unique view as shown in Figure 10.

In addition to live Internet streaming, HDEV is commonly used as supplemental video for ISS daily updates on NASA $\mathrm{TV}$, and can be accessed through NASA Apps on smart phones, and tablets, and over-the-top TV streaming devices such as Roku, and Apple TV 4.

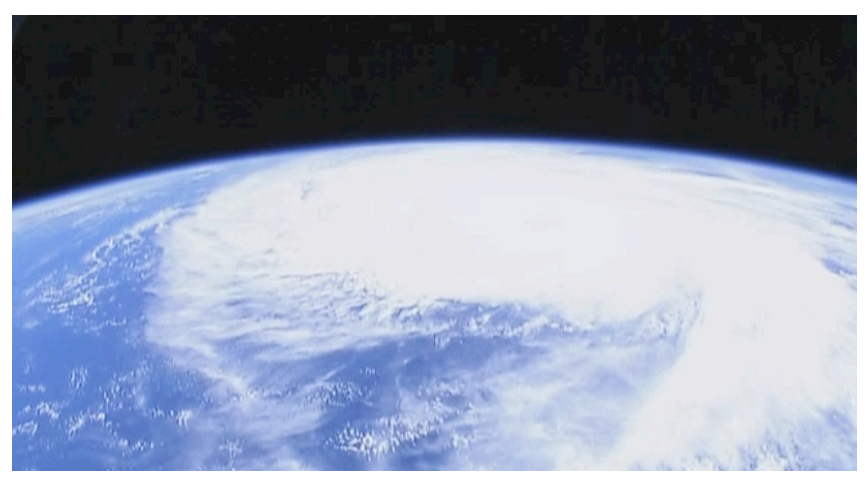

Figure 10. HDEV view of category 5 Hurricane Patricia, 2015

\section{Conclusions ANd Future Work}

A relatively small amount of pixel need corrected since the payload was active. Total resolution is 1.3-2 megapixel [8], thus the Pixel results conclude that the COTS cameras and enclosure performed very well for the period of performance. From the results, both a CCD or CMOS sensor can resist radiation for periods of years in an enclosure such as HDEV's. The radiation resistance could also depend on the camera's angle in the payload enclosure.

If bandwidth is available, future work includes increasing the bandwidth to $8 \mathrm{Mbps}$ to see if there is any appreciable increase in video quality, and perhaps a visible increase in pixel damage due to less compression. To increase the accuracy of determining bad pixels, it would be helpful to have adjustable camera settings such as aperture, zoom, and pan, and tilt.

\section{HDEV's Future}

HDEV was originally planned decommission in 2016, when the Atomic clock EnsEmblE in spAcE (ACES) payload would replace HDEV on the Columbus module's mounting 
location. However, the ACES payload flight hardware has been delayed for shipment. If current schedule holds fro ACES, the total operating time for HDEV in orbit will be about 3 years.

While HDEV is still active, a new HDEV streaming encoder planned for installation for higher resolution Internet streaming, and more avenues planned for viewers to watch, such as Facebook live.

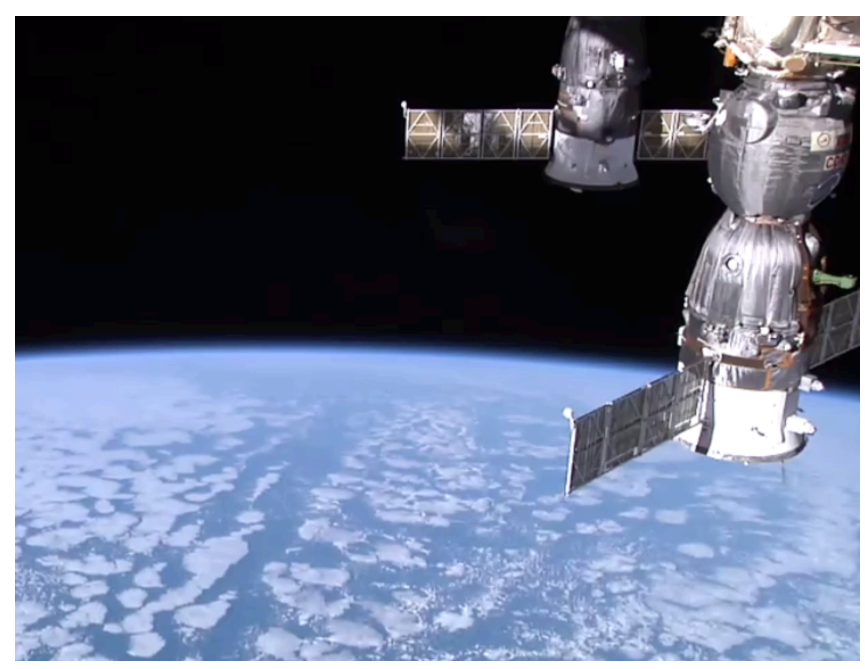

Figure 11. HDEV view of Soyuz capsule

New External High Definition Cameras (EDHCs)

In 2016, new external HD cameras were installed in the ISS known as the external high definition cameras (EDHC). The payloads received astronaut EVA to install [9], but did, in fact, include pan/tilt capability.

The payload cameras consisted of Nikon D4 still cameras operating in video mode inside an enclosure. The camera provides LiveView mode with low data rate imagery provided through Ethernet port [10]. This allows operators to view still or video imagery before downlink.

The EDHC camera and avionics enclosures are similar to HDEV in that they are both custom-built boxes that contain all of the components and protect them from the space environment, with an optical quality window. Just like HDEV, the EDHC enclosure contains dry nitrogen at atmospheric pressure. The enclosure contains a COTS video encoder, Ethernet switch, antennas, and wireless transceiver. The payload enclosure also has pressure, temperature, voltage, and current sensors reporting health back to the ground.

One of biggest differences is that the external HD cameras have full zoom, iris control, and focus control, and they mount on the existing External Television Camera Group (ETVCG) pan/tilt units so ISS has much greater control over the imagery.

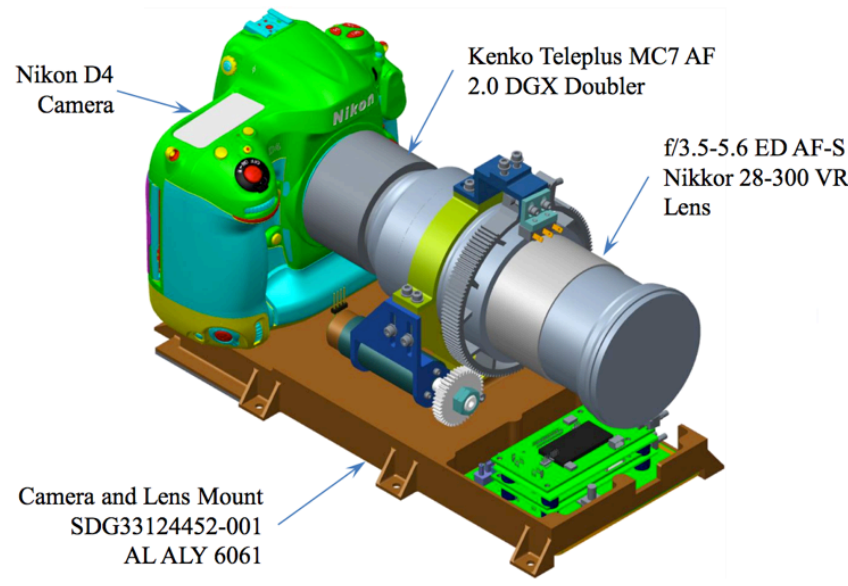

\section{Figure 12. External High Definition Camera enclosure} model Credit: NASA Images [10]

Using Nikon D4 cameras also allows acquisition of full resolution still images if required. The EHDC assembly also uses the VSI AVN-443 encoder, and it is configured just like the ISS internal HD video. It is encoded as an h.264 video stream, $720 \mathrm{p} 8 \mathrm{Mbps}$. The video is downlinked via the $\mathrm{Ku}$ transmitting unit as one of the standard 6-downlink channels. Any still images taken by the external cameras are downlinked via the Joint Station LAN and are transmitted to an imagery server at JSC.

\section{REFERENCES}

[1] Steven-Boniecki, Dwight (2010). Live TV From the Moon. Burlington, Ontario: Apogee Books. ISBN 978-1926592-16-9.

[2] Runco, S. (2015). International Space Station - High Definition Earth Viewing (HDEV), Retrieved from http://www.nasa.gov/mission_pages/station/researc h/experiments/917.html (last access: 2016-10-02).

\section{[3] Optical Payloads for Space Missions By Shen-En Qian}

[3] Rienow A, Graw V, Heinemann S, Schultz J, Selg F, Menz G. Earth observation from the ISS Columbus Laboratory - an open education approach to foster geographical competences of pupils in secondary schools. Living Planet Symposium 2016, Prague, Czech Republic; 2016 May 9-16 7 pp.

[4] Runco, S. (2016). High Definition Earth Viewing (HDEV) Payload Ground Software User's Guide, Houston. August 2016 Edition

[5] Schneider, M. \& Lapenta, C. C. (2001). Payload Operations Integration Center Remote Operations Capabilities. Conference and Exhibit on International Space Station Utilization - 2001, Cape Canaveral, FL, Oct. 15-18, 2001, 1-10. 
[6] (2014). NASA HD earth from space video webcasts, Retrieved from http://www.space.com/25797-nasa-hd-earthfrom-space-video-webcasts.html (last access: 2016-10-02).

[7] (2014). The Ustream NASA HDEV streaming channel. Retrieved from http://www.ustream.tv/channel/iss-hdevpayload (last access: 2016-10-20)

[8] Runco, S. (2015), International Space Station - High Definition Earth Viewing (HDEV), Retrieved from https://eol.jsc.nasa.gov/ESRS/HDEV/

[9] (2016). US EVA 37 successfully completed on ISS, Retrieved from http://spaceflight101.com/iss-expedition48/us-eva-37-successfully-completed-on-iss/ (last access: 2016-10-02)

[10] V. Studer (2014). International Space Station (ISS) External High Definition Camera Assembly (EHDCA), InSpace Inspection Technology Workshop, July 16, 2014 Avionic Systems Division Retrieved from https://ntrs.nasa.gov/archive/nasa/casi.ntrs.nasa.gov/201400 09959.pdf (last access: 2016-10-02).

\section{BIOGRAPHY}

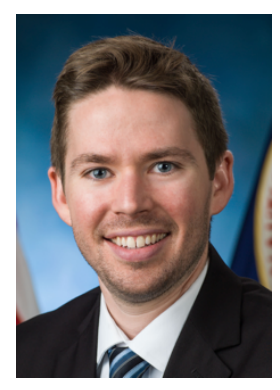

Paul Muri serves as NASA Johnson Space Center's engineering and video operations subject matter expert. Paul received his $B S, M S$, and PhD in Electrical Engineering from University of Florida. While at the university Paul researched in the Wireless and Mobile Systems lab. As a NASA Space Technology Research Fellow, he demonstrated how Delay Tolerant Networking protocols increases video throughput in satellite networks. Paul presented results at conferences in San Francisco, DC, MIT, New York, Google, and Shanghai. Paul has interned for Motorola, Schlumberger, and NASA Goddard Space Flight Center. After earning his PhD, Paul worked for Boeing Satellite Systems in Los Angeles, designing satellite payload and ground communication systems for critical reviews, new business proposals, and global ITU regulatory spectrum affairs.

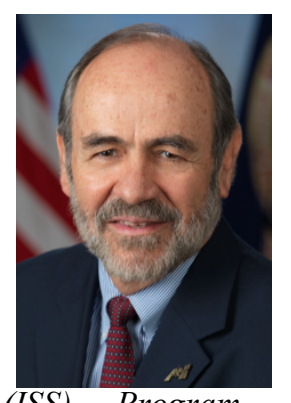

Carlos Fontanot has spent forty years in the imagery field photography, film, television, and broadcasting. After graduating from the University of Houston, he started his professional career in a local television station as Director / Producer of live programs. Currently, he manages imagery for NASA's International Space Station (ISS) Program, including acquisition, downlink, processing, distribution and archiving of digital still images and television. One of his main duties is to integrate ISS imagery requirements from scientists, international partners and the NASA community and ensure that the images are acquired. Carlos defined the basic concept for the High Definition Earth Viewing experiment, which became the foundation for the design and manufacturing of the payload. He is the Co-Principal Investigator for the on board experiment. Fluent in three languages, Carlos was assigned to Moscow, Russia, during the early days of the ISS to establish live broadcasting links for key events and serve as Public Affairs Officer in charge of press conferences and media events leading to the launch of the first crew to live onboard the ISS. One of Carlos' highest priorities in live is the extensive dissemination of space imagery for the benefit of all humankind and to share the breathtaking views of our Planet - Off the Earth for the Earth.

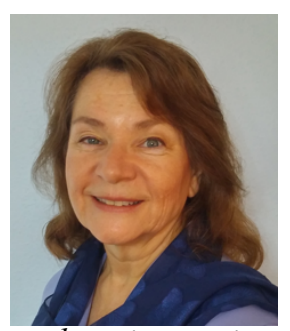

Susan Runco is currently the Deputy Manager, XI4\ARES Exploration Science Office and the Science Operations Co-PI for the ISS High Definition Earth Viewing Experiment on the ISS. She has worked with training astronauts on observing and imaging the Earth, and testing various remote sensing cameras and tools in space since 1988. Prior to NASA, she served in the U.S. Navy, where her duties included being Operations Officer of the Naval Survey Vessel USNS Chauvenet (T-AGS 29), where she planned and conducted hydrographic and oceanographic surveys of the Makassar Strait, Indonesia in 1980/1981. She received an MS (Meteorology and Oceanography) degree from the Naval Postgraduate School, Monterey, CA (June 1986) and a BS degree in Biological/Chemical Oceanography from Florida Institute of Technology (June, 1976).

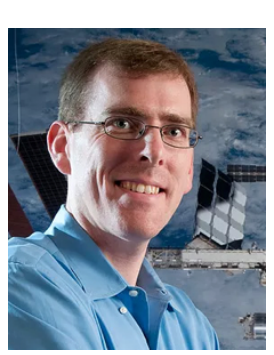

Chris Getteau is an Imagery Specialist and Graphic Designer supporting the International Space Station Mission and Program Integration Contract, and one of the lead designers and project integrators for the High Definition Earth Viewing Experiment. He has 12 years of experience integrating imagery projects for the ISS Program, ranging from small productions to large-scale infrastructure upgrades. In addition to his integration work, Chris has produced mission-related videos, infographics, and posters, and currently works with the Payloads Office to support ISS science outreach. A graduate of Texas Tech University, Chris received a bachelor's degree in Photocommunications in 2001. 